\title{
20 OPEN QUESTIONS ABOUT DEFORMATIONS OF COMPACTIFIABLE MANIFOLDS
}

\author{
EDOARDO BALLICO, ELIZABETH GASPARIM, AND FRANCISCO RUBILAR
}

\begin{abstract}
Deformation theory of complex manifolds is a classical subject with recent new advances in the noncompact case using both algebraic and analytic methods.

In this note we recall some concepts of the existing theory and introduce new notions of deformations for manifolds with boundary, for compactifiable manifolds, and for $q$-concave spaces. We highlight some of the possible applications and give a list of open questions which we intend as a guide for further research in this rich and beautiful subject.
\end{abstract}

\section{Contents}

1. Motivation 1

2. Submersions 2

3. Deformations of manifolds with boundary 3

3.1. Deformations and the $h$-cobordism theorem 4

4. Deformations of compactifiable manifolds $\quad 7$

4.1. Nontrivial examples in dimension one 10

5. Deformations of $q$-concave spaces 11

6. Final remarks 17

7. Acknowledgements 18

$\begin{array}{ll}\text { References } & 18\end{array}$

\section{Motivation}

There exists a beautiful and successful theory of deformations of complex structures for compact manifolds, as described in [Ko] (see also [Mn, H2]). Here we will discuss some aspects of deformation theory of noncompact manifolds. A short recent survey paper in this area appears in [GR]. Many applications to mathematical physics require the use of noncompact manifolds, and some of these were our initial motivation to consider noncompact spaces. For example, in 4 dimensions, the Nekrasov instanton partition function is defined over noncompact spaces and uses equivariant integration, which would evaluate trivially to zero if considered over compact spaces. Similarly, in 6 dimensions the theory of BPS states considers integration over moduli of bundles on noncompact threefolds. There are also various purely mathematical reasons why one might need deformations of complex structures of noncompact manifolds. We are especially interested in toric Calabi-Yau threefolds, and these must necessarily be noncompact. Furthermore, motivated by questions coming from mirror symmetry, we also wish to consider total spaces of 
cotangent bundles and some of their deformations such as noncompact semisimple adjoint orbits. These are just a few among many reasons to develop deformation theory on the noncompact case.

Nevertheless, compact complex manifolds (or compact complex spaces) have several good properties, which noncompact ones lack. For the deformation theory of compact complex manifolds as in the papers by Kodaira and Spencer more than 65 years ago compactness was essential to get global solutions through the use of elliptic PDE's. In all other approaches to deformation theory, even for singular complex spaces, compactness is crucial for deciding existence questions. Thus, we may expect to find additional difficulties when dealing with the noncompact case.

There are many possible choices of how to go about generalizing deformation theory. Here we propose some new concepts, giving examples. Our main goal here is to motivate other researchers to think about some of the beautiful problems on deformation theory, and to such end we will state several open questions which we hope shall serve as motivation.

We will consider deformations of manifolds with boundary, compactifiable manifolds and $q$-concave manifolds. For each of these classes of spaces, we introduce notions of deformations that are interesting to study, and state open questions about them. We also include a section where we relate deformations with cobordisms and we propose a potential new approach to study both themes in a common setting.

\section{Submersions}

The classical Ehresmann fibration theorem says that if $f: M \rightarrow N$ is a proper submersion between smooth manifolds (possibly with boundary), then it is a smooth fiber bundle; hence smoothly locally trivial. The holomorphic analogue is totally false and deformation theory might be described as the study of this failure.

Let us first note that if a holomorphic map is a smooth submersion then it is also a holomorphic submersion. We now formulate this precisely. Consider a holomorphic map $f: X \rightarrow Y$ between complex manifolds. Fix $x \in X$ and set $y:=f(x)$. Let $n$ (resp. $m$ ) be the dimension of $X$ at $x$ (resp. of $Y$ at $y$ ). Let $T_{x} X$ (resp. $T_{y} Y$ ) denote the tangent space at $x$ (resp. $y$ ) of the complex manifold $X$ (resp. $Y$ ). Then $T_{x} X$ (resp. $T_{y} Y$ ) is a complex vector space of dimension $n$ (resp. $m$ ). Let $\Theta_{x} X$ denote the real tangent space of $X$ at $x$ (resp. $Y$ at $y$ ) seen as a $2 n$-dimensional (resp. $2 m$-dimensional) differentiable manifold. We have $\operatorname{dim}_{\mathbb{R}} \Theta_{x} X=2 n, \operatorname{dim}_{\mathbb{R}} \Theta_{y} Y=2 m$,

$$
\Theta_{x} X \otimes_{\mathbb{R}} \mathbb{C} \cong T_{x} X \oplus \overline{T_{x} X} \text { and } \Theta_{y} Y \otimes_{\mathbb{R}} \mathbb{C} \cong T_{y} Y \oplus \overline{T_{y} Y},
$$

where $\bar{V}$ denote the complex conjugate of the complex vector space $V$.

Lemma 1. If $f: X \rightarrow Y$ is a submersion at $x$ of differentiable manifolds, i.e. if the real differential $\vartheta_{x}: \Theta_{x} X \rightarrow \Theta_{y} Y$ is surjective, then $f$ is a submersion at $x$ as complex manifolds, i.e. the $\mathbb{C}$-linear map $d f_{x}: T_{x} X \rightarrow T_{y} Y$ is surjective.

Proof. The surjectivity of the real differential $\vartheta_{x}: \Theta_{x} X \rightarrow \Theta_{y} Y$ implies the surjectivity of the $\mathbb{R}$-linear map $\vartheta_{x \otimes \mathbb{C}}: \Theta_{x} X \otimes_{\mathbb{R}} \mathbb{C} \rightarrow \Theta_{y} Y \otimes_{\mathbb{R}} \mathbb{C}$, i.e. of 
the map $d f_{x} \oplus \overline{d f_{x}}: T_{x} X \oplus \overline{T_{x} X} \rightarrow T_{y} Y \oplus \overline{T_{y} Y}$. To prove our claim it is sufficient to prove that the real rank of $d f_{x} \oplus \overline{d f_{x}}$ is twice the complex rank of $d f_{x}$. Fixing a basis, we can see the linear map $d f_{x} \oplus \overline{d f_{x}}$ as a diagonal matrix. Denote by $J$ the complex Jacobian of $f$, since we are assuming $f$ holomorphic, denoting the real Jacobian of $f$ by $J_{\mathbb{R}}$ we have

$$
J_{\mathbb{R}}=\left[\begin{array}{ll}
J & 0 \\
* & \bar{J}
\end{array}\right]
$$

Since $\vartheta_{x}$ is surjective, the rank of $J_{\mathbb{R}}$ is $2 n$. So we have

$$
2 n=\operatorname{rk}(J)+\operatorname{rk}(\bar{J})=2 \operatorname{rk}(J),
$$

hence real rank of $d f_{x} \oplus \overline{d f_{x}}$ is twice the complex rank of $d f_{x}$. Therefore, $J$ has rank $n=\operatorname{dim} T_{y} Y$ which is maximal, thus we conclude that $f$ is a submersion as complex manifolds.

Example 2. Here is a classic example of a holomorphic submersion that is not holomorphically locally trivial. Let $S=\mathbb{C} \backslash\{0,1\}$, and consider the family $X$ of elliptic curves in $\mathbb{P}^{2}$ parametrized by $\lambda \in S$ given by

$$
y^{2} z=x(x-z)(x-\lambda z) .
$$

Then the corresponding proper submersion

$$
\pi: \begin{array}{ccc}
X & \rightarrow & S \\
(\lambda,[x: y: z]) & \mapsto & \lambda
\end{array}
$$

is certainly not locally trivial because its fibers are not isomorphic.

\section{Deformations of MANifolds With BOUNDARY}

Fix $r \in(\mathbb{N} \backslash\{0\}) \cup\{+\infty\}$. Let $M$ and $N$ be Hausdorff $C^{r}$-manifolds with a countable basis for their topology. Let $C^{r}(M, N)$ denote the set of all $C^{r}$-maps $f: M \rightarrow N$. There are two useful topologies (the weak and the strong topology) on the set $C^{r}(M, N)$ [Hi, Ch. 2, §1], which however are the same when $M$ is compact [Hi, last 4 lines of page 35]. Since in this section we consider manifolds with boundary, we only need the case in which $M$ is compact, thus we describe the weak topology (also called the compact-open topology for $f$ and its derivatives). Let $(\varphi, U)$ and $(\psi, V)$ be charts on $M$ and $N$ respectively. Let $K \subset U$ be a compact set such that $f(K) \subset V$. Fix a positive real number $\varepsilon$ and a non-negative integer $k$. Let | | denote the euclidean norm on $\psi(V)$. Let $\mathcal{N}(f ;(U, \varphi),(V, \psi), \varepsilon, k)$ denote the set of all $g \in C^{r}(M, N)$ such that

$$
\left|D^{i}\left(\psi f \varphi^{-1}\right)(x)-\left(\psi g \varphi^{-1}\right)(x)\right|<\varepsilon
$$

for all $x \in \varphi(K)$ and all partial derivatives $D^{i}$ of order $i \leq k$.

The weak topology on $C^{r}(M, N)$ is the topology for which the collection of all sets $\mathcal{N}(f ;(U, \varphi),(V, \psi), \varepsilon, k)$ is prescribed to be a subbasis, i.e. an open set for this topology is an arbitrary union of a finite intersection of sets $\mathcal{N}(f ;(U, \varphi),(V, \psi), \varepsilon, k)$. In the remaining of this section we use the weak topology on $C^{r}(M, N)$.

There is also the notion of $C^{r}$ manifold with a boundary $(M, \partial M)$ and a topology for the family of all $C^{r}$-maps $C^{r}((M, \partial M) ;(N, \partial N))$ between two manifolds with boundaries [Hi, Ch. 2,§3]. We use these notions to define a 
$C^{r}$-locally trivial family of complex manifolds with boundary. However, as in [Hi, pp. 30-31] we may also consider maps from a manifold $(M, \partial M)$ with $\partial M \neq \emptyset$ to a manifold $Y$ with $\partial Y=\emptyset$.

Definition 3. Let $X, Y$ be $C^{\infty}$ manifolds and $f: X \rightarrow Y$ a $C^{\infty}$-map. We say that $f$ is a deformation of connected $n$-dimensional complex manifolds if:

- $X$ and $Y$ are connected, and $\operatorname{dim} X=\operatorname{dim} Y+n$ with $n>0$

- $f$ is a submersion with connected fibres $\left\{M_{t}\right\}_{t \in Y}$

- there exists a countable open covering $\left\{U_{i}\right\}_{i \geq 1}$ of $X$ such that for each integer $i \geq 1$ there exists a collection of $n C^{\infty}$ functions

$$
z_{i}^{1}(q), \ldots, z_{i}^{n}(q): U_{i} \rightarrow \mathbb{C}
$$

such that for each $t \in Y$, the assignment

$$
\left\{q \mapsto\left(z_{i}^{1}(q), \ldots, z_{i}^{n}(q)\right)_{\mid U_{i} \cap f^{-1}(t)}\right\}
$$

provides a complex structure on $M_{t}$.

Remark 4. Contrary to [Ko, p. 184] we do not assume that $f$ is proper, i.e. we do not assume that each $M_{t}$ is compact.

Definition 5. We say that $f$ is $C^{\infty}$ locally trivial on the base if for each $t \in Y$ there is an open neighborhood $U$ of $t \in Y$ and a diffeomorphism $v: f^{-1}(U) \rightarrow U \times M_{t}$ such that $\pi_{1} \circ v=f_{\mid f^{-1}(U)}$, where $\pi_{1}: U \times M_{t} \rightarrow U$ is the projection onto the first factor.

Next we recall the definition of deformation that appears in [GR].

Definition 6. [GR, Def. 9] A deformation family of a complex manifold $X$ is a holomorphic surjective submersion $\tilde{X} \stackrel{\pi}{\rightarrow} D$, where $D$ is a complex disc centred at 0 (possibly a vector space, possibly infinite dimensional), satisfying:

- $\pi^{-1}(0)=X$

- $\tilde{X}$ is locally trivial in the $C^{\infty}$ category.

The fibres $X_{t}=\pi^{-1}(t)$ are called deformations of $X$.

In further generality, allowing the parameter space to be a variety or a scheme, and requiring that the bundle be locally trivial, we obtain the concept of a family of complex structures.

Remark 7. Note that in Def. 3 we are not assuming that $f$ is locally trivial in the $C^{\infty}$-category. Def. 3 is not the same that [GR, Def. 9]. Indeed, [GR, Ex. 7] shows that these two definitions are not equivalent in full generality, but we propose the following question.

Question 1. Under what conditions we can say that the two notions of deformations mentioned above are equivalent, or one implies the other?

3.1. Deformations and the $h$-cobordism theorem. The fundamental idea behind cobordism theory is that 2 manifolds are cobordant if their disjoint union forms the boundary of some manifold. Since a manifold $M$ and any of its deformations $M^{\prime}$ occur in a family, by considering the family 
restricted to a path on the base connecting the fibers $M$ to $M^{\prime}$, we see that these are cobordant.

In this section, we suggest a relation between deformations of complex manifolds with boundary and the very well known $h$-cobordism theorem. This is just one of many possible connections among the concepts of deformations and cobordisms. We quote from the introduction of Stong [St]: As in any subject, the primary problem is classification of the objects within isomorphism and determination of effective and computable invariants to distinguish the isomorphism classes. In our setup, the notion of cobordant manifolds can be regarded as such a computable invariant.

It is worth mentioning that in various setups (but not all) deformation equivalent implies diffeomorphic, therefore homeomorphic. So, one might wish to use the notions of diffeomorphic or homeomorphic manifolds instead of cobordant. However, it is well known that deciding if 2 smooth manifolds are homeomorphic is not computable in the following precise sense: It has been known for a long time that for any finitely presented group $S$, it is possible to construct a 4 dimensional real manifold $M(S)$ with fundamental group $S$ in such a way that $M(S)$ and $M(R)$ will be homeomorphic if and only if $S$ and $T$ are isomorphic groups. However, the word problem of deciding when 2 finitely presented groups are isomorphic is unsolvable [Ma]. Cobordism, being a milder invariant, is more computable.

We recall some basic ideas and fundamental results about cobordisms and refer the readers to the beautiful book by Stong [St] and references therein for the general theory.

Definition 8. An $(n+1)$-dimensional cobordism is a quintuple $(W ; M, N, i, j)$ consisting of an $(n+1)$-dimensional compact differentiable manifold with boundary $W$; closed $n$-manifolds $M, N$ and embeddings $i: M \hookrightarrow \partial W$ and $j: N \hookrightarrow \partial W$ with disjoint images such that

$$
\partial W=i(M) \sqcup j(N) .
$$

There are many types of cobordisms such as: oriented, unoriented, framed, real, complex, relative, unitary, special unitary, symplectic, Lagrangian, exact, $h$-cobordisms, s-cobordisms, and so on. Here, the choice of to focus on $h$-cobordisms simply reflects the preference of the second author when typing this paper, but certainly many other types are of interest. Without making any attempt to review the vast literature on the subject, we just point out one example of application of the concept complex cobordisms, which follows from the more general result for weakly complex manifolds [Mi1, Thm.1"] proved by Milnor and Novikov:

Proposition 9. If a complex manifold is the boundary of another complex manifold, then all of its Chern numbers are zero.

We now discuss $h$-cobordisms. For a classical introduction to this subject, we suggest [Mi1, Mi2]. A brief overview can be found in the $h$-cobordism wikipedia page https://en.wikipedia.org/wiki/H-cobordism.

Definition 10. An $(n+1)$-dimensional cobordism $(W ; M, N, i, j)$ is an $h$-cobordism if the inclusions $i: M \hookrightarrow \partial W$ and $j: N \hookrightarrow \partial W$ are homotopy equivalences. 
We recall some fundamental and well known results in low dimensions concerning $h$-cobordisms and then, passing to dimension at least 5 , we state the $h$-cobordism theorem, proved in 1962 by S. Smale [S2] expanding on ideas of his proof (just one year earlier) of the Poincaré conjecture in dimension greater than or equal to 5 [S1].

For $n=0$, the $h$-cobordism theorem is trivially true: the interval is the only connected cobordism between connected 0-manifolds.

For $n=1$, the $h$-cobordism theorem is vacuously true, since there is no closed simply-connected 1-dimensional manifolds.

For $n=2$, the $h$-cobordism theorem is equivalent to the Poincaré conjecture stated by Poincaré in 1904 (one of the Millennium Problems) and was proved by Grigori Perelman in [P1, P2, P3].

For $n=3$, the $h$-cobordism theorem for smooth manifolds has not been proved and, due to the 3-dimensional Poincaré conjecture, is equivalent to the hard open question of whether the 4-sphere has non-standard smooth structures.

For $n=4$, the $h$-cobordism theorem was proved by Freedman and Quinn for topological manifolds [FQ], but the analogous statement in the PL and smooth categories was shown to be false by Donaldson [D].

The following is one of the most famous theorems in cobordism theory:

Theorem 11 (h-cobordism). Let $n$ be at least 5 and let $W$ be a compact $(n+1)$-dimensional $h$-cobordism between $M$ and $N$ in the category $\mathcal{C}=$ Diff, PL, or Top such that $W, M$ and $N$ are simply connected, then $W$ is $\mathcal{C}$-isomorphic to $M \times[0,1]$. The isomorphism can be chosen to be the identity on $M \times\{0\}$.

This means that the homotopy equivalence between $M, W$, and $N$ is homotopic to a $\mathcal{C}$-isomorphism.

There ought to be many restrictions to families of deformations in complex dimensions 3 and higher coming from this result, which apply to deformations of complex structures, and are certainly worth studying. In this initial study, we just list some very basic questions.

Question 2. Compare the notions of cobordism and deformations of compact manifolds.

Cobordisms themselves may be considered in family and such families appear in the recent literature considered within perspectives of various types of geometry and topology such as [IJP, p. 136] and [ALP, p. 61] as well as in current research in symplectic geometry, in particular in connection to Floer theory [WW] and Fukaya categories of Lagrangian cobordisms [BC, C].

Question 3. Compare the notion of deformations of manifolds with boundaries with the notion of families of cobordisms.

Question 4. Let $n \geq 2$. If an $n$-dimensional complex manifold $M$ with boundary has nontrivial deformations, then what type of cobordism exists between the components of $\partial M$ ?

If the assumption that $M$ and $N$ are simply connected is dropped, $h$-cobordisms need not be cylinders; the obstruction is exactly the Whitehead torsion $\tau(W, M)$ of the inclusion $M \hookrightarrow W$. 
There exits a stronger version of the $h$-cobordism theorem, known as the $s$-cobordism theorem. Namely, the $s$-cobordism theorem (the $s$ stands for simple-homotopy equivalence), proved independently by Barry Mazur, John Stallings, and Dennis Barden, states (with the same assumptions as above but where $M$ and $N$ need not be simply connected):

Theorem 12 (s-cobordism). An h-cobordism is a cylinder if and only if Whitehead torsion $\tau(W, M)$ vanishes.

The torsion vanishes if and only if the inclusion $M \hookrightarrow W$ is not just a homotopy equivalence, but a simple-homotopy equivalence.

Note that one need not assume that the other inclusion $N \hookrightarrow W$ is also a simple homotopy equivalence, it follows from the theorem.

Considering when $h$-cobordisms are trivial, we start our approach towards the main question considered in Q. 20 concerning deformation theory in the non-compact case. Meanwhile, returning to deformation theory, recall that the core results of Kodaira-Spencer theory state that for a compact complex manifold $X$, the first cohomology group with coefficients in the tangent sheaf, that is $\mathrm{H}^{1}(X, T X)$, parametrizes infinitesimal deformations of $X$, with corresponding obstructions given by elements of $\mathrm{H}^{2}(X, T X)$. In the case of cobordisms, the Whitehead torsion characterizes when an $h$-cobordism is a cylinder. Thus, it is natural to consider the following question.

Question 5. Is there a numerical invariant that characterizes when a manifold with boundary is rigid, similarly to the way Whitehead torsion characterizes when an $h$-cobordism is a cylinder?

Categorically, the collection of $h$-cobordisms forms a groupoid. Then a finer statement of the $s$-cobordism theorem is that the isomorphism classes of this groupoid (up to $\mathcal{C}$-isomorphism of $h$-cobordisms) are torsors for the respective Whitehead groups $\mathrm{Wh}(\pi)$, where $\pi \cong \pi_{1}(M) \cong \pi_{1}(W) \cong \pi_{1}(N)$.

Question 6. Express the definitions and properties of deformations of manifolds with boundaries in the language of groupoids and torsors.

\section{Deformations of COMPaCtifiable manifolds}

When we are only interested in an open subset $X$ of a compact complex manifold $W$ such that $D:=W \backslash X$ is a hypersurface, we may consider deformations of pairs $(W, D)$ such as in [I]. This may be too restrictive for the purpose of holomorphic deformations when we are only interested in $X$, even if we know that $X$ can be compactified; such a notion has been considered in the literature [Ka], and here we give a slightly generalized version. In our definition of compactifiable deformation of the pair $(X, W)$ we do not require that the map be a submersion (nor that it be flat) at the points of $D$. We inquire how restrictive are our assumptions and we pose a few questions related to the compactifiability of deformations of the threefolds $W_{k}$ studied in [BGS, GKRS].

Definition 13. Take finitely many holomorphic functions $f_{1}, \ldots, f_{k}$ in a domain $D$ of $\mathbb{C}^{n}$ and form their ideal sheaf

$$
\mathcal{I}:=\mathcal{I}_{D}:=\mathcal{O}_{D} f_{1}+\cdots+\mathcal{O}_{D} f_{k} \subset \mathcal{O}_{D}
$$


The quotient sheaf $\mathcal{O}_{D} / \mathcal{I}_{D}$ is a sheaf of rings on $D$. Put

$$
X:=\operatorname{Supp}\left(\mathcal{O}_{D} / \mathcal{I}_{D}\right)=\left\{x \in D: \mathcal{I}_{D, x} \neq \mathcal{O}_{D, x}\right\}, \quad \text { and } \quad \mathcal{O}_{X}:=\left.\left(\mathcal{O}_{D} / \mathcal{I}_{D}\right)\right|_{X} .
$$

Clearly, $X$ equals the set

$$
N(\mathcal{I}):=N\left(f_{1}, \ldots, f_{k}\right)=\left\{x \in D: f_{1}(x)=\cdots=f_{k}(x)=0\right\}
$$

of common zeros of $f_{1}, \ldots, f_{k}$ in $D$ and, moreover, $X$ is closed in $D$. Every stalk $O_{X, x}=\left(\mathcal{O}_{D, x} / \mathcal{I}_{x}\right)$ is an analytic algebra, hence $\mathcal{O}_{X}$ is a sheaf of local $\mathbb{C}$-algebras on $X$. The $\mathbb{C}$-ringed space $\left(X, \mathcal{O}_{X}\right)$ is called the (complex) model space, defined (on $D$ ) by $\mathcal{I}$. We write $V\left(f_{1}, \ldots, f_{k}\right)$ or simply $V(\mathcal{I})$ for this space. Note that $|V(\mathcal{I})|=N(\mathcal{I})$.

Definition 14. A $\mathbb{C}$-ringed space $\left(X, \mathcal{O}_{X}\right)$ is called a complex space, if $X$ is a Hausdorff space and if every point of $X$ has an open neighborhood $U$ such that the open $\mathbb{C}$-ringed subspace $\left(U, \mathcal{O}_{U}\right)$ of $\left(X, \mathcal{O}_{X}\right)$ is isomorphic to a complex model space. Thus, locally, complex spaces are determined by finitely many holomorphic functions in domains of number spaces.

Complex model spaces are complex spaces. In a complex space $\left(X, \mathcal{O}_{X}\right)$ every open set $U \subset X$ defines an open complex subspace $\left(U, \mathcal{O}_{U}\right)$. Complex spaces form a (full) subcategory of the category of $\mathbb{C}$-ringed spaces. Morphisms (isomorphisms) are called holomorphic (biholomorphic) maps; $\mathcal{O}_{X}$-modules on a complex space $\left(X, \mathcal{O}_{X}\right)$ are called analytic sheaves on $X$.

Algebraic properties of the analytic algebras $\mathcal{O}_{X, x}$ are used to introduce geometrical notions. For instance, we say that smooth points are those points $x \in X$ for which $\mathcal{O}_{X, x}$ is regular, i.e. isomorphic to a $\mathbb{C}$-algebra $\mathbb{C}\left\{z_{1}, \ldots, z_{n}\right\}$. Complex spaces with smooth points only are called complex manifolds; standard examples are domains in $\mathbb{C}^{n}$ and Riemann surfaces. Non smooth points are called singular points; simple examples of singular points are the origin of Neil's parabola $y=a x^{3 / 2}, a \neq 0$ constant, and the origin of the cone $w^{2}-z_{1} z_{2}=0$ in $\mathbb{C}^{3}$.

Definition 15. Let $X$ be a complex space. We say that $X$ is compactifiable if there are a compact complex space $X^{\prime}$, a closed analytic subset $D \subset X^{\prime}$ and an open embedding $j: X \rightarrow X^{\prime}$ such that $j(X)=X^{\prime} \backslash D$. If in addition we can take $X^{\prime}$ to be a projective variety with $X$ embedded analytically on $X^{\prime}$ we call it a projectivizable deformation of $X$.

Note that in definition 15 we do not fix the pair $\left(X^{\prime}, D\right)$. Note also that requiring an algebraic embedding instead of an analytic one would result in a different definition. By Hironaka desingularization if $X$ is a smooth compactifiable complex space, then we may find a compactification $\left(X^{\prime}, D, j\right)$ with $X^{\prime}$ smooth and $D$ with normal crossings. Note also that by definition quasi-projective varieties are compactifiable, but not conversely.

Definition 16. Let $X$ be compactifiable complex space. A compactifiable deformation of $X$ is a sextuple $(\mathcal{X}, D, Y, f, o, j)$, where:

- $\mathcal{X}$ and $Y$ are complex spaces,

- $D$ is a closed analytic subset of $\mathcal{X}$ and $o \in Y$,

- $f: \mathcal{X} \rightarrow Y$ is a proper holomorphic map such that $f_{\mid \mathcal{X} \backslash D}$ is flat, 
- $j: X \rightarrow f^{-1}(o)$ is an open embedding with

$$
j(X)=f^{-1}(o) \backslash D \cap f^{-1}(o) .
$$

We say $(\mathcal{X}, D, Y, f, o, j)$ is topologically trivial along $X$ if

$$
f_{\mathcal{X} \backslash D}: \mathcal{X} \backslash D \rightarrow Y
$$

is topologically locally trivial over $Y$, i.e. for each $u \in Y$ there is an open neighborhood $A$ of $u$ such that $f_{(\mathcal{X} \backslash D) \cap f^{-1}(A)}:(\mathcal{X} \backslash D) \cap f^{-1}(A) \rightarrow A$ and the projection $(\mathcal{X} \backslash D) \cap f^{-1}(A) \times A \rightarrow A$ are isomorphic as maps of topological spaces over $A$.

When $X$ is smooth, we say that $(\mathcal{X}, D, Y, f, o, j)$ is a smooth compactifiable deformation if $f_{\mid \mathcal{X} \backslash D}$ is a smooth morphism of complex spaces and each fibre of $f$ is a complex manifold. If $\mathcal{X}$ can be chosen to be a projective variety we say that the deformation is projectivizable.

For a smooth compactifiable deformation we do not assume that $Y$ is a complex manifold. This is the main reason to use topological local triviality in Definition 16 instead of differential triviality. The interested reader may do the definition for differential local triviality when $Y$ is smooth.

Example 17. Let $X$ be a connected compact manifold of dimension $n>0$. Let $S \subset X$ be a nonempty finite set and $U=X \backslash S$. Let $(\mathcal{X}, D, Y, f, o, j)$ be a smooth compactifiable deformation of $X$. Since $f$ is proper and the deformation of $X$ is smooth, all fibres of $f$ are compact manifolds with deformations of $U$ as Zariski open subsets. Restricting $Y$ to a smaller neighborhood of $o$ if needed, we may assume that all fibres of $f$ are connected $n$-dimensional complex manifolds and $f_{\mid D}: D \rightarrow Y$ is a finite holomorphic map. Thus all fibres of $f_{\mid D}$ are finite sets. It is easy to check that $(\mathcal{X}, D, Y, f, o, j)$ is topologically trivial along $X$ if and only if all fibres of $f_{\mid D}$ have the same number of points.

Question 7. Let $X^{\prime}$ be a compactification of the complex space $X$. When are deformations of $X$ obtained from deformations of $X^{\prime}$ and deformations of the analytic set $D:=X^{\prime} \backslash X$ ?

We now discuss a few basic examples.

Example 18. Let $C$ be a smooth and connected complex curve of genus $g \geq 0$. Take as $D$ a finite set, $X:=C \backslash D$. Set $m:=\sharp(D)$. In such a case deformations of $X$ topologically trivial over the base always come from deformations of $C$. If either $g \geq 2$, or $g=1$ and $m>0$, or else $g=0$ and $m \geq 3$, then we are just speaking about the deformation theory of pairs $(C, D)$, which is the (well-known) deformation theory of elements of $\mathcal{M}_{g, m}$.

Example 19. Let us a consider the following noncompact surfaces

$$
Z_{k}=\operatorname{Tot}\left(\mathcal{O}_{\mathbb{P}^{1}}(-k)\right) \text {. }
$$

That is, total spaces of line bundles on $\mathbb{P}^{1}$. In [BG1, Thm. 5.4 and Thm.6.18], the authors prove that these surfaces admit smooth compactifiable deformations. In fact, [GKRS, Lem. 5.6] show that these deformations can be obtained from deformations of the well known Hirzebruch surfaces

$$
\mathbb{F}_{k}=\operatorname{Proj}\left(\mathcal{O}_{\mathbb{P}^{1}}(-k) \oplus \mathcal{O}_{\mathbb{P}^{1}}(0)\right),
$$


which are compact.

Example 20. The noncompact threefolds

$$
W_{k}=\operatorname{Tot}\left(\mathcal{O}_{\mathbb{P}^{1}}(-k) \oplus \mathcal{O}_{\mathbb{P}^{1}}(k-2)\right)
$$

admit deformations which are not smooth compactifiable. In [GKRS, Thm. 6.3] and [BG1, Cor.1.29] there are concrete examples of infinite dimensional families of deformations of $W_{k}$ for $k \geq 2$ which are not smooth compactifiable. Further, in [BGS, Cor. 1.25] the authors show that deformations of $W_{k}$ are not obtained from their compactifications. This situation is quite different from the one of the surfaces $Z_{k}$ considered in the previous example.

Question 8. Determine which deformations of $W_{k}$ are compactifiable.

Question 9. Describe all projectivizable deformations of $W_{k}$.

Question 10. Decide whether there exist nontrivial deformations of $W_{1}$. Note that [GKRS] proved that $W_{1}$ is formally rigid.

Question 11. Consider a compactifiable (or projectivizable) deformation $(\mathcal{X}, D, Y, f, o, j)$ of $X=W_{k}$. For which $t \in Y$ is there a surjection $f^{-1}(t) \rightarrow$ $\mathbb{P}^{1}$ extending the structure of $\mathcal{X} \cap f^{-1}(t)$ as an affine bundle over $\mathbb{P}^{1}$ ? For which $t \in Y$ is $f^{-1}(t)$ at least covered by codimension 1 projective spaces?

Question 12. Does there exist a compactifiable space $X$ such that no nontrivial deformation of $X$ is compactifiable?

Question 13. Let $X$ be a toric Calabi-Yau threefold. Determine what conditions on $X$ imply that all of its deformations are compactifiable (if any).

4.1. Nontrivial examples in dimension one. To give some very nontrivial examples, we recall some definitions and results from [E].

Definition 21. Let $f: X \rightarrow Y$ be a holomorphic submersion between complex manifolds. $f$ is said to be differentially locally trivial over $Y$ (resp. locally trivial over $Y$ ) if for each $y \in Y$ there is an open neighborhood $U$ of $y$ such that the map $f_{\mid f^{-1}(U)}: f^{-1}(U) \rightarrow U$ is differentially (resp. holomorphically) isomorphic to the projection $f^{-1}(y) \times U \rightarrow U$.

If the submersion $f$ is proper, then it is differentially locally trivial over $Y$ by the classical theorem of Ehresmann. As mentioned in the introduction, the complex analogue is false. Furthermore, the theorem of Fischer and Grauert [FG] says that if the fibers are compact connected complex manifolds, then $f$ is holomorphically locally trivial if and only if all the fibers $f^{-1}(y)$ are analytically isomorphic for all $y \in Y$. The hypothesis of compact fibers is essential here, as [GR, Ex. 7] demonstrates.

Theorem 22. [E, Thm. III] If $X$ is a smooth surface, $Y$ a smooth curve, all fibres of $f$ are biholomorphic to an algebraic curve $F$ and $f$ is compactifiable, then $f$ is locally trivial over $Y$.

We recall that a connected one-dimensional complex manifold $F$ is of finite type if and only if there is a compact complex one-dimensional manifold $T$ and a holomorphic embedding $j: F \rightarrow T$ such that $\overline{j(F)} \backslash F$ is topologically 
the union of finitely many disjoint circles and points. The following very strong result is due to $\mathrm{K}$. Yamaguchi:

Theorem 23. [Y, Thm.3] Let $f: W \rightarrow Y$ be a holomorphic submersion with $Y$ a one-dimensional complex manifold, $W$ a Stein surface and all fibres of $f$ biholomorphic to the same connected one-dimensional complex manifold with finite type $F$. Assume that $F$ is neither biholomorphic to the open disc nor to the open disc minus its center. Then $f$ is holomorphically a locally trivial fibration over $Y$.

Thus here one does not need to assume that the submersion is compactifiable, but $[Y]$ requires a very strong assumption (Steinness) on the total space of the holomorphic submersion $f$. We just recall the following example from [E]. Other examples of nontrivial holomorphic fibrations are contained in $[\mathrm{Y}]$ and the references quoted therein.

Example 24. [E, Ex. 1.2.1] Set $T:=\{(0,0)\} \cup\left\{\left(z, z^{-1}\right)\right\}_{z \in \mathbb{C}^{*}} \subset \mathbb{C}^{2}$. Set $X:=\mathbb{C}^{2} \backslash T$. Let $f: X \rightarrow \mathbb{C}$ denote the restriction to $X$ of the projection of $\mathbb{C}^{2}$ onto its factors. All fibres of the submersion $f$ are biholomorphic to $\mathbb{C}^{*}$, but $f$ is neither differentiably nor topologically locally trivial over $\mathbb{C}$. Note that $X$ is not Stein.

\section{Deformations of $q$-COnCave spaces}

We use the notation from [HL]. For extensions of these definitions to complex spaces, see for instance [A3, Ch. 5]. Let $X$ be a connected complex manifold of dimension $n$ and $x \in X$. Then $X$ is a $2 n$-dimensional differentiable manifold and we call $T_{\mathbb{R}, x} X$ its real tangent space, which is a $2 n$-dimensional real vector space. Set

$$
T_{\mathbb{C}, x} X:=T_{\mathbb{R}, x} X \otimes \mathbb{C} .
$$

Thus $T_{\mathbb{C}, x} X$ is a $2 n$-dimensional complex vector space. If $z_{1}, \ldots, z_{n}$ are local coordinates of $X$ around $x$, we may take $\frac{\partial}{\partial z_{1}}, \ldots, \frac{\partial}{\partial z_{n}}, \frac{\partial}{\partial \bar{z}_{1}}, \ldots, \frac{\partial}{\partial \bar{z}_{n}}$ as a basis of $T_{\mathbb{C}, x} X$. Let $T_{x}^{\prime} X$ (resp. $T_{x}^{\prime \prime} X$ ) be the subspace of $T_{\mathbb{C}, x} X$ spanned by $\frac{\partial}{\partial z_{1}}, \ldots, \frac{\partial}{\partial z_{n}}$ (resp. $\left.\frac{\partial}{\partial \bar{z}_{1}}, \ldots, \frac{\partial}{\partial \bar{z}_{n}}\right)[\mathrm{HL}, \mathrm{p} .59]$. If $Y \subset X$ is a real $C^{1}$ submanifold of $X$ we set $T_{x}^{\prime} Y:=T_{x}^{\prime} X \cap T_{\mathbb{C}, x} Y$.

Definition 25. Let $\rho: X \rightarrow \mathbb{R}$ be a $C^{2}$ function. For any $x \in X$ we define a Hermitian form $L_{\rho}(x)$ on $T_{x}^{\prime} X$, called the Levi form of $\rho$, in the following way. If $\left(t_{1}, \ldots, t_{n}\right) \in \mathbb{C}^{n}$ and $t=\sum_{j=1}^{n} t_{j} \frac{\partial}{\partial z_{j}}$ set

$$
L_{\rho}(x)(t):=\sum_{j, k=1}^{n} \frac{\partial^{2} \rho(x)}{\partial \bar{z}_{j} \partial \bar{z}_{k}} \bar{t}_{j} t_{k} .
$$

The signature (also called the inertia) of the Levi form at $x$ does not depend on the choice of the holomorphic local coordinates $z_{1}, \ldots, z_{n}$, see [HL, pp. 59-60], [A3, p.61].

Definition 26. Fix an integer $q$ such that $1 \leq q \leq n$. We say that a $C^{2}$ function $\rho$ is $q$-convex if at all $x \in X$ the Levi form of $\rho$ has at least $q$ positive eigenvalues [HL, p. 60] and that it is $q$-concave if $-\rho$ is $q$-convex, i.e. if at all points of $X$ the Levi form of $\rho$ has at least $q$ negative eigenvalues [HL, p. 73]. 
A $C^{2}$-function $\rho$ is called pseudoconvex (resp pseudoconcave) if it is ( $n-$ $1)$-convex (resp. $(n-1)$-concave). The $n$-convex functions are those usually called strictly plurisubharmonic.

Definition 27. Let $\rho: X \rightarrow \mathbb{R}$ be a $C^{2}$ function. Set $\beta:=\sup _{z \in X} \rho(z) \in$ $\mathbb{R} \cup\{+\infty\}$. The function $\rho$ is called an exhausting function if there is a compact subset $K \subseteq X$ such that $\rho(x)<\beta$ for all $x \in X \backslash K$ and for all $c<\beta$ the set $\{z \in X \mid \rho(z)<c\}$ is relatively compact in $X$ [HL, I.5.1].

The exhausting function $\rho$ is called $q$-concave at infinity if there is a compact $K \subset X$ with $X \backslash K \neq \emptyset$ and such that $\rho_{\mid X \backslash K}$ is $q$-concave.

Definition 28. We say that an $n$-dimensional complex manifold $X$ is $q$-concave (resp. $q$-convex) for $0 \leq q \leq n-1$, if there exists an exhausting function of $X$, which is $(q+1)$-concave (resp. $q$-convex) at infinity [HL, p. 73, p. 65].

Now assume that $X$ is a connected open subset of the connected complex manifold $W$. Fix $q \in\{1, \ldots, n-1\}$. In [HL, pp. 138-139] it is defined when $W$ is a $q$-concave extension of $X$. We recall the basic idea of the definition, referring the reader to [HL] for technical details. A $q$-concave extension in $X$ is defined locally in domains, in the same spirit as the manifold is obtained by local charts. The choices of domains $V \subset X$ are typically quite delicate and require satisfying a series of rather technical conditions.

Definition 29. Assuming a domain $V \subset X$ has been appropriately chosen, consider $A_{1} \subset A_{2}$ such that $A_{2} \backslash A_{1} \subset V$. We say that $A_{2}$ is a $q$-concave extension of $A_{1}$ in $X$ provided there exists a local chart $\psi: V \rightarrow U \subset \mathbb{C}^{n}$ such that $U$ is a $q$-concave domain in $\mathbb{C}^{n}$ and there exists a $q+1$-concave function $\rho: U \rightarrow \mathbb{R}$ of class $C^{2}$ such that $\psi\left(A_{2} \backslash A_{1}\right)=\{z \in U: \rho(z)<0\}$ is relatively compact in $U$ and forms a $q$-concave configuration in $\mathbb{C}^{n}$ (see [HL, Def. 13.1]).

Intuitively, we may think of a $q$-concave manifold as being concave in $q$ dimensions, and similar for $q$-convex. Concave and convex extensions can be thought of as being represented by the following pictorial situation. Suppose a physical space $X$ is an extension of a proper subspace $D \subset X$ and an observer is positioned in $X \backslash D$, then:

(Fig.1) If $X$ is a concave extension of $D$, then the observer sees $D$ as concave. (Fig.2) If $X$ is a convex extension of $D$, then the observer sees $D$ as convex.

Note however that the notions of $q$-concave and $q$-convex extensions of $D$ are concepts referring to a "small" neighborhood of $D$ and do not imply that $X$ is either concave or convex itself, as the pictures show.

Remark 30. Let $W$ be a connected complex manifold that is a $q$-concave extension of its connected open subset $X \subset W$. Assume that $X$ is $q$-concave. Then the restriction map $H^{i}(W, T W) \rightarrow H^{i}(X, T X)$ is an isomorphism for $i \leq q-1$ by [HL, IV.15.11] and is injective if $i=q$ by [HL, IV.16.1], since the Dolbeault and the usual cohomology groups coincide by [HL, Thm. I.2.14].

Definition 31. An $n$-dimensional $(n-1)$-concave complex manifold is called a pseudoconcave complex manifold. 


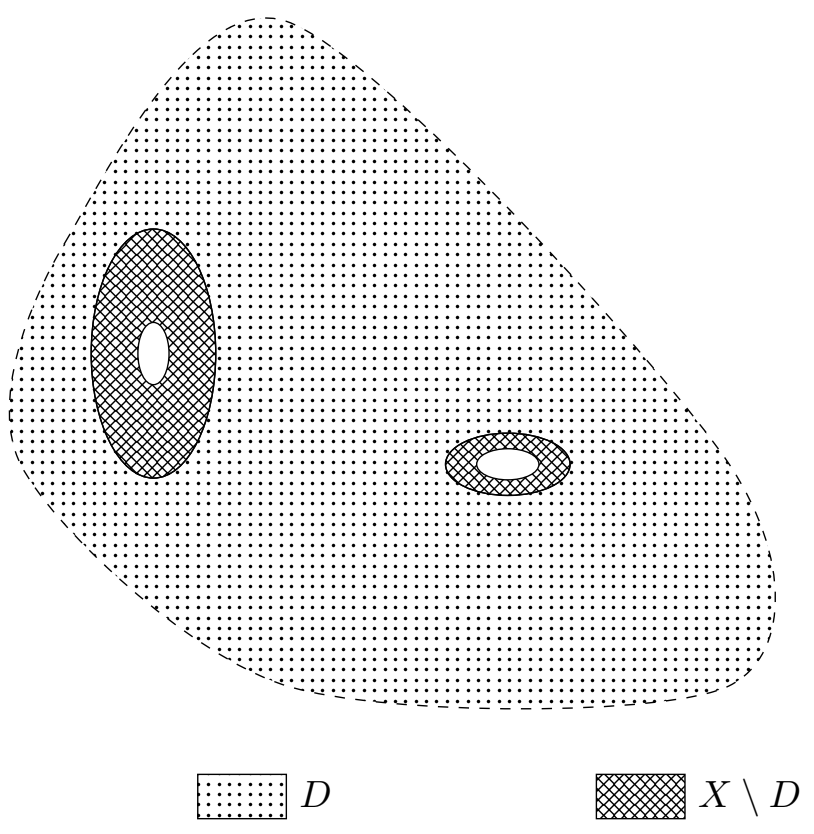

Figure $1 . X$ is a $q$-concave extension of $D$.

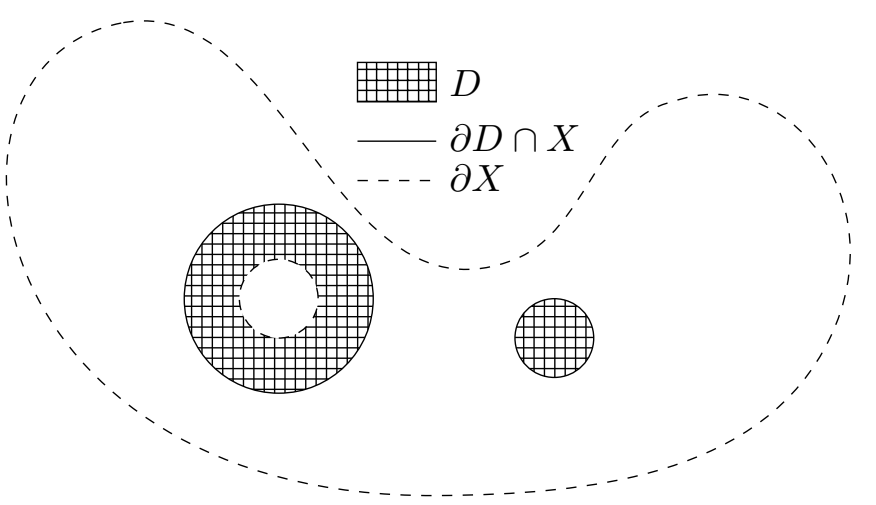

Figure 2. $X$ is a $q$-convex extension of $D$.

Example 32. Every compact complex space is trivially pseudoconcave. More examples are easily obtained by taking "convex holes" in a compact space. The compactification problem (solved by H. Rossi) says that if a space $X$ is pseudoconcave and $\operatorname{dim} X \geq 3$, then $X$ is an open subset of a compact space (it may be singular) [HL, §23] which is an $(n-1)$-concave extension of $X$, hence one can "fill in" convex holes in $\operatorname{dim} \geq 3$.

Example 33. Not every 2-dimensional pseudoconcave space is compactifiable. Indeed, [A3, Ex. 3] shows how to construct a pseudoconcave structure on $\mathbb{P}^{2} \backslash\{p\}$ which is not compactifiable. 
Thus, it is not very restrictive (but restrictive) to only look at $q$-concave complex manifolds with a compact $q$-concave extension. Here we take a pair $(W, X)$ with $X q$-concave, $W$ a compact complex manifold and we discuss smooth $q$-concave deformations. For simplicity, in what follows we assume that the parameter space $Y$ is smooth and also that the total space of the deformation $\mathcal{X}$ is smooth.

Definition 34. A $q$-concave deformation of the pair $(W, X)$ is a quintuple $(\mathcal{X}, \mathcal{W}, Y, o, f)$, satisfying:

- $Y$ is a complex space, and $o \in Y$,

- $f: \mathcal{W} \rightarrow Y$ is a proper submersion,

- $\mathcal{X}$ is an open subset of $\mathcal{W}$,

- $\left(f^{-1}(o), f^{-1}(o) \cap \mathcal{X}\right)$ is biholomorphic to the pair $(W, X)$, and

- $f^{-1}(y)$ is a $q$-concave extension of $f^{-1}(y) \cap \mathcal{X}$ for each $y \in Y$.

The last requirement means that $f_{\mid \mathcal{X}}$ is transversally $q$-concave, that is, $\mathcal{W}$ is a $q$-concave extension of $\mathcal{X}$ in which the data of the $q$-extension is transversal to $f$.

When $f_{\mid \mathcal{X}}$ is topologically locally trivial, we say that the deformation is topologically locally trivial over $Y$. When $Y$ is a smooth manifold and $f_{\mid \mathcal{W}}$ is differentially locally trivial over $Y$ we say that the deformation is differentially locally trivial over $Y$.

Now assume $q \geq 3$. By Remark 30 the restriction map

$$
H^{i}(W, T W) \rightarrow H^{i}(X, T X)
$$

is an isomorphism for each $i \leq 2$ and is an isomorphism in degree 3 if $q>3$. Since these are the cohomologies used in Kodaira-Spencer theory, it then follows that of $q \geq 3$ the deformation theory of $W$ is governed by data on $X$. For instance, if $H^{1}(X, T X)=0$, then in any $q$-concave deformation $(\mathcal{X}, \mathcal{W}, Y, o, f), \mathcal{W}$ is locally trivial over $Y$ and hence we just ask how to deform $X$ inside $W$ (if we only want $q$-concave deformations of $(W, X)$ ).

Take a $q$-concave deformation $(\mathcal{X}, \mathcal{W}, Y, o, f)$ of the pair $(W, X)$ with $Y$ smooth and let $E$ be a holomorphic vector bundle on $X$. By [RR, Thm. 3 $\S 8$ p. 123] there is a range of integer $i \in \mathbb{N}, q$ and $\operatorname{dim} Y$ for which the direct images $R^{i} f_{*}(E)$ are coherent. However, to study deformations, we only need the case $E=T \mathcal{X}$ and in this case $E$ is the restriction to $\mathcal{X}$ of the vector bundle $T \mathcal{W}$. Since $f: \mathcal{W} \rightarrow Y$ is proper, Grauert theorem of coherence for proper maps says that for all $i \in \mathbb{N}$ the $\mathcal{O}_{Y^{-}}$-sheaf, $R^{i} f_{*}(T \mathcal{W})$ is coherent, each cohomology group $H^{i}\left(f^{-1}(y), T f^{-1}(y)\right)$ is finite-dimensional and the function $y \mapsto \operatorname{dim} H^{i}\left(f^{-1}(y), T f^{-1}(y)\right)$ is an upper semicontinuous function $Y \rightarrow \mathbb{N}$. We then obtain the following result:

Proposition 35. Let $(W, X)$ be a $q$-concave pair, $q \geq 3$. Then all $q$-concave deformations $(\mathcal{X}, \mathcal{W}, Y, o, f)$ of $(W, X)$ with $Y$ smooth are obtained from deformations of $X$.

Proof. Using Remark 30 we have

$$
\operatorname{dim} H^{i}\left(f^{-1}(y), T f^{-1}(y)\right)=\operatorname{dim} H^{i}\left(f^{-1}(y) \cap \mathcal{X}, T f^{-1}(y) \cap \mathcal{X}\right)
$$

for all $i \leq q-1$. 
Let $X$ be an $n$-dimensional connected complex space, $n \geq 3$. For simplicity we assume here that $X$ is smooth and relegate to Remark 39 the case of singular complex spaces. Assume that $X$ is pseudoconcave, i.e. that it is $(n-1)$-concave [A2, A3, HL].

Remark 36. Even for projective manifolds $X$ it is classically known (e.g. K3 surfaces, i.e. a compact 2-dimensional Calabi-Yau manifold) that small deformations of $X$ may not be projective.

The usual way to remain in the category of projective manifolds is not just to consider deformations of $X$, but instead to consider deformations of a pair $(X, L)$, where $L$ is an ample line bundle on $X$. In this section we will do the same for pseudoconcave complex manifolds. We follow [A3, AS, AT].

Assume the existence of a line bundle $L$ on $X$ such that for each $x \in X$, there is a positive integer $m$ and $f_{1}, \ldots, f_{n} \in H^{0}\left(X, L^{\otimes m}\right)$ such that $f_{i}(x)=$ 0 for all $i$ and (after taking a local trivialization of $L$ around $x), f_{1}, \ldots, f_{m}$ induce a local biholomorphism of $X$ near $x$ with $\mathbb{C}^{n}$ near 0 . When $n=2$ one needs also to assume that for all $x, y \in X$ such that $x \neq y$ there is a positive integer $m$ and $f \in H^{0}\left(X, L^{\otimes m}\right)$ such that $f(x)=0$ and $f(y) \neq 0$. Under these assumptions the $\mathbb{C}$-algebra

$$
A(X, L):=\oplus_{m \geq 0} H^{0}\left(X, L^{\otimes m}\right)
$$

is finitely generated and hence the scheme

$$
\Gamma:=\operatorname{Proj}(A(X, L)
$$

is a well-defined complex projective scheme [H1]. Since $L$ is a line bundle on $X$ and it is known that for each $x \in X$ there is a positive integer $m$ and $f \in H^{0}\left(X,{ }^{\otimes m}\right)$ with $f(x) \neq 0$, the pair $(X, L)$ induces a holomorphic map

$$
u: X \rightarrow \Gamma \text {. }
$$

Then $X$ is an integral complex space (for manifolds it just means that $X$ is connected). Since $X$ is smooth, it is a normal complex space [Fi, Thm. p. 120]. Let $v: W \rightarrow \Gamma$ be the normalization of $\Gamma$ as a complex space $[\mathrm{Fi}, \S 2.26]$, which is just the complex space associated to the normalization of $\Gamma$ as an integral algebraic variety. We get that $u$ factors as

$$
u=v \circ j \quad \text { with } \quad j: X \rightarrow W
$$

[Fi, pp.121-122]. It is proved in [A3, AS, AT] that $j$ is an open embedding and in the following we will identify $j$ with the identity map, i.e. we will see $X$ as an open subset of the normal projective complex space $W$. Examples contained in the quoted paper show that for $X$ smooth $W$ need not be smooth, but it has at most finitely many singular points.

By the construction of $\Gamma$ as a Proj there exists a natural line bundle on it and its pull-back $R$ to $X$ has the property that it is ample and $R_{\mid X}=L$. Taking instead of $L$ a power $L^{\otimes m}, m \gg 0$, we may assume that $R$ induces an embedding of $W$ into a large projective manifold. Using the definition of projective morphisms between two complex spaces [BS2, Ch. 4] we may define projective deformations of the pair $(X, R)$ in the following way. Take $W$ and $R$ as above. 
Definition 37. A projective deformation of $(X, L, W, R)$ is given by $(\mathcal{W}, Y, f, o, \mathcal{X}, \mathcal{R})$ such that:

- $\mathcal{W}$ and $Y$ are complex spaces,

- $o \in Y$ and $f^{-1}(o)=W$,

- $f: \mathcal{W} \rightarrow Y$ is a holomorphic submersion which is projective in the sense of [BS2, Ch.4],

- $\mathcal{X}$ is an open subset of $\mathcal{W}$,

- $f_{\mid \mathcal{X}}: \mathcal{X} \rightarrow Y$ is pseudoconcave,

- $f^{-1}(o) \cap \mathcal{X}=X$,

- $\mathcal{R}$ is a line bundle on $\mathcal{W}$ which is ample with respect to $f$ and

- $\mathcal{R}_{\mid f^{-1}(o)} \cong R$.

Note that by definition $f$ is projective if and only if there is a line bundle on $\mathcal{W}$ which is ample with respect to $f$ [BS2, p. 159].

Remark 38. Assume $n \geq 3$ and hence that $W$ is a 2-concave extension of $X$. Assume that $W$ is smooth and that $H^{1}(T X)=0$. Since $H^{1}(W, T W)=$ $H^{1}(X, T X), W$ is rigid. We get a kind of rigidity for pseudoconcave deformations of the pair $(X, L)$ : the only nearby pseudoconcave deformations are as open subsets of $W$ for the following reason. Since $W$ is rigid, any small deformation of $W$ is biholomorphic to the projection $\pi_{2}: W \times \Delta \rightarrow \Delta$ with $\Delta$ an open neighborhood of $0 \in \mathbb{C}^{N}$, some $N \geq 0$. By the definition of pseudoconcave deformation such a small deformation is obtained taking an open subset $\mathcal{X}$ of $W \times \Delta$ such that $\mathcal{X} \cap \pi_{2}^{0}=W$ and $\pi_{2 \mid \mathcal{X}} \mathcal{X} \rightarrow \Delta$ is a submersion. Thus, this submersion is equivalent to taking a family $\left\{\pi_{2}^{-1}(z) \cap \mathcal{X}\right\}_{z \in \Delta}$ of open subsets of $W$ with $\pi_{2}^{-1}(0)=X$.

Now we may take as a pseudoconcave deformation of $(X, L)$ any family with $\mathcal{X}$ an open subset of $\mathcal{W}$ such that $\mathcal{X} \cap f^{-1}(o)=X$ and $f_{\mid \mathcal{X}}$ is pseudoconcave.

Remark 39. For general background on singular complex spaces, see [Fi]. See $[\mathrm{Fi}, \S 2.6,2.7]$ for the differential of a holomorphic map between arbitrary complex spaces and in particular for the notion of submersion between complex spaces [Fi, §2.18]. For the notions of $q$-convexity and $q$-concavity for complex spaces, see for instance $[\mathrm{A} 3, \S 5.2]$

Question 14. Let $X$ be a pseudoconcave complex space. When is there a compact complex space $W$ and an open embedding $j: X \rightarrow W$ such that $W \backslash j(X)$ is "very small", for instance a finite set?

One could think of Question 14 as a particular case of the following vague question.

Question 15. Let $X$ be a complex space. Find an object, say a compact topological space, $\bar{X}$ containing $X$ with $X$ open in $\bar{X}$ and for which certain sequences (or all sequences) of points of $X$ have a subsequence converging to a point of $\bar{X}$, then give a structure of complex space to $\bar{X}$. Classify those complex spaces $X$ such that $\bar{X} \backslash X$ is small, e.g. it is a finite set.

Such a program was carried out for noncompact one-dimensional complex manifolds in several ways, using non-holomorphic data, like the existence or non-existence of certain potential [RS, pp.20-21], [AS], at least if $\pi_{1}(X)$ 
is finitely generated then giving several different criteria which determine when $\bar{X} \backslash X$ is small.

When $n:=\operatorname{dim} X \geq 2$ and $X$ is smooth, a related notion is the one of envelope of holomorphy, in which we assume that $X$ has many nonconstant holomorphic functions and the aim is to find $\bar{X}$ which is Stein. This very old problem (see [JP] for the case of Riemann domains) is discussed in details in [DSST, §11.7.3, §13.7].

We consider here the case of a compact projective $W$ which is normal with only finitely many singular points (this is similar to [AT, case (b) of Proposition 6]). Then we expect that to verify when $W \backslash j(X)$ is finite should not be very difficult, although we do not have an obvious criterion to see it. We have:

Proposition 40. Let $X$ be an n-dimensional pseudoconcave complex manifold. Assume that one of the following conditions is satisfied:

(1) either $n \geq 3$ and for each $x \in X$ there exists an integer $t>0$ and $f_{1}, \ldots, f_{t} \in \omega_{X}^{\otimes t}$ giving local coordinates at $x$; or

(2) for each $x \in X$ there exists an integer $t>0$ and $f_{1}, \ldots, f_{n} \in \omega_{X}^{\otimes t}$ giving local coordinates of $X$ at $x$ and for all $x, y \in X$ such that $x \neq y$ there exists $m>0$ and $f \in H^{0}\left(\omega_{X}^{\otimes m}\right)$ such that $f(x)=0$ and $f(y) \neq 0$.

Then there exists a normal projective variety $W$ and an open embedding $j: X \rightarrow W$.

Proof. Part (2) is [AT, Thm.2] or [A3, Thm.4.3.1]. Part (1) follows from part (2) and [A3, Thm. 4.4.1].

We then expect that the following instance of the vague question phrased in 15 should be solvable in reasonably short time.

Question 16. Solve question 15 for spaces $X$ satisfying the properties in Proposition 40.

\section{FinAl REMARKS}

We have stated a few concrete open questions on deformation theory of noncompact manifolds, of compactifiable manifolds, manifolds with boundary, and $q$-concave spaces. Each of the sections 3, 4, and 5 may give rise to an entire graduate thesis, and certainly to new publications. Also, each question about deformations has a corresponding question about moduli which should also be addressed. Moreover, there are many large fundamental questions about deformations that remain open, we finish by stating a few of them.

Question 17. Under what conditions do all deformations of the total space of a vector bundle have the structure of affine bundles?

Question 18. Let $X$ be a noncompact Calabi-Yau threefold. What geometric conditions on $X$ imply that it has infinitely many nonisomorphic deformations? (Compare with [BGS] and [GKRS]).

Question 19. Let $X$ be obtained from $\tilde{X}$ by removing finitely many points. Compare deformations of $X$ to those of $\tilde{X}$. 
Finally, perhaps the question that must be answered most urgently:

Question 20. What is the correct cohomology theory that parametrizes deformations of noncompact manifolds? (Vanishing of such a cohomology should imply existence of only trivial deformations.)

\section{Acknowledgements}

This worked started during a meeting of Research in Pairs supported by the Centro Internazionale per la Ricerca Matematica, of Fondazione Bruno Kessler, Trento (Italy). F. R. was supported by Beca Doctorado Nacional Conicyt Folio 21170589. E. G. and F. R. thank the Office of External Activities of ICTP for the support under Network grant NT8. E.B. was partially supported by MIUR and GNSAGA of INdAM (Italy). The authors thank Severin Barmeier for suggesting several improvements to the original text. Finally, we gladly acknowledge the help of the referee, who made us not only improve the paper, but also learn more about the subject.

\section{REFERENCES}

[ALP] M. Abreu, F. Lalonde, L. Polterovich, New perspectives and challenges in symplectic field theory, CRM Proc. \& Lecture Notes 49, Am. Math. Soc., 2009.

[AS] L. V. Ahlfors and L. Sario, Riemann Surfaces, Princeton University Press, Princeton, NJ, 1960.

[A2] A. Andreotti, Théorèmes de dépendance algébrique sur les espaces complexes pseudo-concaves, Bull. Soc. Math. France 91 (1963), 1-38.

[A3] A. Andreotti, Nine lectures on complex analysis, in: Complex Analysis, Edizioni Cremonese, Rome (1974) 1-175.

[AS] A. Andreotti and Y.-T. Siu, Projective embedding of pseudoconcave spaces, Ann. Sc. Norm. Sup. Pisa, s. 3, 24 (1970), 231-278.

[AT] A. Andreotti and G. Tomassini, Some remarks on pseudoconcave manifolds, Essays in topology and related topics, Mémoires dédiés à G. de Rham, Springer-Verlag, Berlin, 1970.

[BGS] E. Ballico, E. Gasparim, B. Suzuki, Infinite dimensionality of deformation families for noncompact threefolds, J. Pure Appl. Algebra 225 no. 4, (2021) 106554, 24pp..

[BG1] S. Barmeier, E. Gasparim, Classical deformations of local surfaces and their moduli of instantons, J. Pure and Applied Algebra, 223 no. 6, (2019), 2543-2561.

[BS2] C. Bănică, O. Stănăşila, Méthodes algébriques dans la théorie globale des espaces complexes, Vol. I. and II, Gauthier-Villars, Paris, 1977.

[B] J. Bingener, Lokale Modulräume in der analytischen Geometrie, Band 1 and 2, Vieweg, Braunsweig/Wiesbaden, 1987.

[BC] P. Biran, O. Cornea, Lagrangian cobordism and Fukaya categories, Geom. Funct. Anal., 24(6) (2014) 1731-1830.

[C] E. Campling, Fukaya categories of Lagrangian cobordisms and duality, arXiv:1902.00930.

[DSST] G. Della Sala, A. Saracco, A. Simioniuc and G. Tomassini, Lectures on complex analysis and analytic geometry, Edizioni della Normale, Pisa, 2006.

[D] S. Donaldson, Irrationality and the h-cobordism conjecture. J. Differential Geom. 26 no.1 (1987) 141-168.

[E] G. Elencwajg, Submersions holomorphes à fibres isomorphes, Fonctions de plusieurs variables complexes III (Sem. François Norguet, 1975-1977), pp. 209-223, Lecture Notes in Math. 670 Springer, Berlin, 1978.

[Fi] G. Fischer, Complex Analytic Geometry, Lect. Notes in Math. 538 Berlin-Heidelberg-New York, 1976. 
[Fs] S. D. Fischer, Function theory of planar domains, John Wiley \& Sons, New York, 1983.

[FG] W. Fischer, H. Grauert, Lokal-triviale Familien kompakter komplexer Mannigfaltigkeiten, Nachr. Akad. Wiss. Göttingen Math.-Phys. Kl. II (1965) 89-94.

[FQ] M. Freedman, F. Quinn, Topology of 4-manifolds. Princeton Mathematical Series. 39. Princeton, NJ: Princeton University Press. 1990.

[GKRS] E. Gasparim, T. Köppe, F. Rubilar, B. Suzuki, Deformations of noncompact Calabi-Yau threefolds, Rev. Colombiana de Matemáticas, 52 no. 1 (2018) 41-57.

[GR] E. Gasparim, F. Rubilar, Deformations of noncompact Calabi-Yau manifolds, families and diamonds, to appear in Contemp. Math..

[I] D. Iacono, Deformations and obstructions of pairs $(X, D)$, Int. Math. Res. Not. 19 (2015) 9660-9695.

[JP] M. Jarnicki, P. Pflug, Extensions of holomorphic functions, Walter de Gruyter, Berlin - New York, 2000.

[H1] R. Hartshorne, Algebraic Geometry, Springer-Verlag, Berlin-Heidelberg-New York, 1977.

[H2] R. Hartshorne, Deformation Theory, Springer, New York, 2010.

[HL] G. M. Henkin, J. Leiterer, Andreotti-Grauert theory by integral formulas, Progress in Math. 74, Birkhäuser, Basel, 1988.

[Hi] M. W. Hirsch, Differential topology, Springer-Verlag, Berlin, 1976.

[IJP] I. Itenberg, B. Jöricke, M. Passare, Perspectives in Analysis, Geometry, and Topology, Progress in Mathematics 296, Birkhauser (2012).

[Ka] Y. Kawamata, On deformations of compactifiable complex manifolds, Proc. Japan Acad. 53 (1977), 106-109.

[Ko] K. Kodaira, Complex manifolds and deformations of complex structures, Grundlehren der mathematischen Wissenschaften 283, Berlin: Springer (1986).

[Mn] M. Manetti, Lectures on Deformations of Complex Manifolds, Rendiconti di Matematica, Ser. VII 24 (2004) 1-183.

[Ma] A. A. Markov, Insolubility of the problem of homeomorphy, Proc. Internat. Congr. Math. (1958) 300-306.

[Mi1] J. Milnor, Survey of cobordism theory, L'Enseignement Mathématique 8 (1962).

[Mi2] J. Milnor, Lectures on the h-cobordism theorem, Princeton Univ. Press, 1965.

[P1] G. Perelman, The entropy formula for the Ricci flow and its geometric applications arXiv:math/0211159.

[P2] G. Perelman, Ricci flow with surgery on three-manifolds arXiv:math/0303109.

[P3] G. Perelman, Finite extinction time for the solutions to the Ricci flow on certain three-manifolds arXiv:math/0307245.

[RR] J. P. Ramis, G. Ruget, Résidus et dualité, Invent. Math. 26 (1974) 89-131.

[RS] B. Rodin; L. Sario, D., Principal functions, Van Nostrand Company, Inc. Princeton, NJ, 1968.

[S1] S. Smale, Generalized Poincaré's conjecture in dimensions greater than four, Ann. of Math. (2) 74 (1961) pp. 391-406.

[S2] S. Smale, On the structure of manifolds, Amer. J. Math. 84 (1962) pp. 387-399

[St] R. E. Stong, Notes on cobordism theory, Princeton Univ. Press and Tokyo Univ. Press, 1968.

[WW] K. Wehrheim, C.T. Woodward, Floer cohomology and geometric composition of Lagrangian correspondences. Adv. Math. 230 (2012) no. 1, 177-228.

[Y] K. Yamaguchi, Famille holomorphe de surfaces de Riemann ouvertes qui est une variété de Stein, J. Math. Kyoto Univ. 16 (1976) no. 3, 497-530.

Ballico - Dept. Mathematics, University of Trento, I-38050 Povo, Italy. Gasparim, Rubilar - Depto. Matemáticas, Universidad Católica del Norte, Antofagasta, Chile.

BALLICO@SCIENCE.UNITN.IT, ETGASPARIM@GMAIL.COM, RUBILAR_N17@HOTMAIL.COM 